\title{
Effects of Different Types of Mechanical Loading on Trabecular Bone Microarchitecture in Rats
}

\author{
Yong-In Ju', Teruki Sone ${ }^{2}$ \\ ${ }^{1}$ Department of Health and Sports Sciences, Kawasaki University of Medical Welfare, Kurashiki, Okayama; \\ ${ }^{2}$ Department of Nuclear Medicine, Kawasaki Medical School, Kurashiki, Okayama, Japan
}

Corresponding author

Yong-In Ju

Department of Health and Sports Sciences, Kawasaki University of Medical Welfare, 288 Matsushima, Kurashiki, Okayama 7010193, Japan

Tel: +81-86-462-1111

Fax: +81-86-464-1109

E-mail: jyi.k051@mw.kawasaki-m.ac.jp

Received: September 3, 2021

Revised: September 28, 2021

Accepted: October 6, 2021
Mechanical loading is generally considered to have a positive impact on the skeleton; however, not all types of mechanical loading have the same beneficial effect. Many researchers have investigated which types of mechanical loading are more effective for improving bone mass and strength. Among the various mechanical loads, high-impact loading, such as jumping, appears to be more beneficial for bones than low-impact loadings such as walking, running, or swimming. Therefore, the different forms of mechanical loading exerted by running, swimming, and jumping exercises may have different effects on bone adaptations. However, little is known about the relationships between the types of mechanical loading and their effects on trabecular bone structure. The purpose of this article is to review the recent reports on the effects of treadmill running, jumping, and swimming on the trabecular bone microarchitecture in small animals. The effects of loading on trabecular bone architecture appear to differ among these different exercises, as several reports have shown that jumping increases the trabecular bone mass by thickening the trabeculae, whereas treadmill running and swimming add to the trabecular bone mass by increasing the trabecular number, rather than the thickness. This suggests that different types of exercise promote gains in trabecular bone mass through different architectural patterns in small animals.

Key Words: Cancellous bone $\cdot$ Jumping $\cdot$ Rats $\cdot$ Running $\cdot$ Swimming

\section{INTRODUCTION}

Physical exercise has generally been considered to have positive impacts on the skeleton, since the mechanical loading generated by muscle contraction and ground reaction force (GRF) stimulate bone formation. Furthermore, physical exercise can help to reduce the risk of falls that could lead to fractures or other injury by building muscle strength and improving balance. However, different types of exercise do not necessarily show equally beneficial effects. Over the last couple of decades, numerous researchers have investigated which types of exercise are more effective for improving bone mass and strength. More specifically, gymnasts, track athletes, soccer, volleyball, and basketball players who participate in weight-bearing and impact sports have higher bone mass than adolescents and adults involved in non-weight-bearing sports such as swimming.[1-5] The notion that high-impact and weight-bearing activity is more effective for bone formation than low- 
impact loading is thus generally accepted. Several animal studies have suggested that high-impact loading such as jumping exercise seems more beneficial for increasing bone mass and strength than low-impact loading such as running exercise.[6,7] These results suggest that higher strain rates occur with jumping activities compared with running, despite similar magnitudes of strain.[8,9] Different types of exercise thus appear to manifest differently in terms of bone adaptation.

Although considerable research has been conducted into mechanical loading and structural changes to the trabecular bone in histomorphometric studies,[6,10-12] the effects are still not completely understood. In particular, almost no research has examined the relationships between different types of exercise and structural characteristics of trabecular bone. Several previous studies using histomorphometric analyses have indicated that the increase in trabecular bone mass with resistance training is primarily due to increased trabecular thickness, whereas trabecular number was unaffected. In contrast, running exercise induced increases in trabecular bone mass by increasing the number of trabeculae. Accordingly, jumping and running exercises might exert different influences on trabecular architecture. We recently tested this hypothesis by comparing the effects of jumping and running exercises on the trabecular architecture of the distal femur in growing rats using high-resolution micro-computed tomography (microCT).[13] The effects on trabecular bone mass were seen to differ between jumping and running exercises. Jumping exercise increased trabecular bone mass mainly by thickening the trabeculae, whereas running exercise resulting in a greater number of trabeculae. These results support the previous finding that in vivo tibial compressive loading and running exercises improved trabecular architecture in the proximal metaphysis in different manners.[14] Considering such findings, different types of exercise appear to have different influences on trabecular bone microarchitecture. Although several studies have reported that different forms of mechanical loading might have different influences on trabecular architecture, this point has barely been discussed. Changes to the trabecular bone structure in animals are discussed in this review, particularly with regard to the 3 different exercises of treadmill running, jumping, and swimming.

\section{CHANGES IN TRABECULAR BONE MICROSTRUCTURE UNDER SKELETAL UNLOADING}

Chronic reductions in mechanical loading such as immobilization, bed rest, spinal cord injury, and exposure to microgravity are well known to precipitate generalized skeletal loss, particularly in bones that bear weight under normal conditions. To simulate and study bone changes induced under microgravity environments, Morey et al. [15] developed a model using tail suspension in 1979. This model of skeletal unloading via tail suspension has also become the gold standard to assess alterations in the skeleton during complete unloading.[16,17] In histomorphometric analyses of proximal tibias isolated from 6-week-old rats, Basso et al. [18] confirmed that 14 days of skeletal unloading by hindlimb suspension resulted in reductions of $50 \%$ to trabecular bone volume fraction, $50 \%$ to trabecular number, and $25 \%$ to trabecular thickness, and an increase of $179 \%$ to trabecular separation in the secondary spongiosa. In previous experiments with 3-dimensional (3D) micro-CT of the distal femoral metaphysis, we have also demonstrated that the decrease in bone volume induced by unloading was primarily caused by a decrease in trabecular number (-51\% reduction) as opposed to a decrease in trabecular thickness (-21\% reduction) for Wistar rats after 2 weeks of tail suspension.[19] Tail-suspended rats exhibited loss of trabeculae, particularly from the central zone of the femur with the loss of trabecular number, although the remaining trabeculae did not show significant differences in thickness compared with the control group (Fig. 1). These results imply that the loss of cancellous bone during 2 weeks of unloading by tail suspension is predominantly due to decreases in trabecular bone number rather than trabecular thickness. This conclusion is supported by the histomorphometric work of Bourrin et al. [20], who demonstrated that the decreased bone volume in the secondary spongiosa of the proximal tibial metaphysis after 2 weeks of tail suspension was mainly attributable to the disappearance of trabeculae (-23\%) rather than a thinning of the trabeculae. Furthermore, similar findings have been confirmed in humans subjected to unloading caused by prolonged bed rest [21] and spinal cord injury.[22] As an example, Modlesky et al. [22] reported that the distal femur and proximal tibia of spinal cord-injured men showed $27 \%$ and $20 \%$ lower 


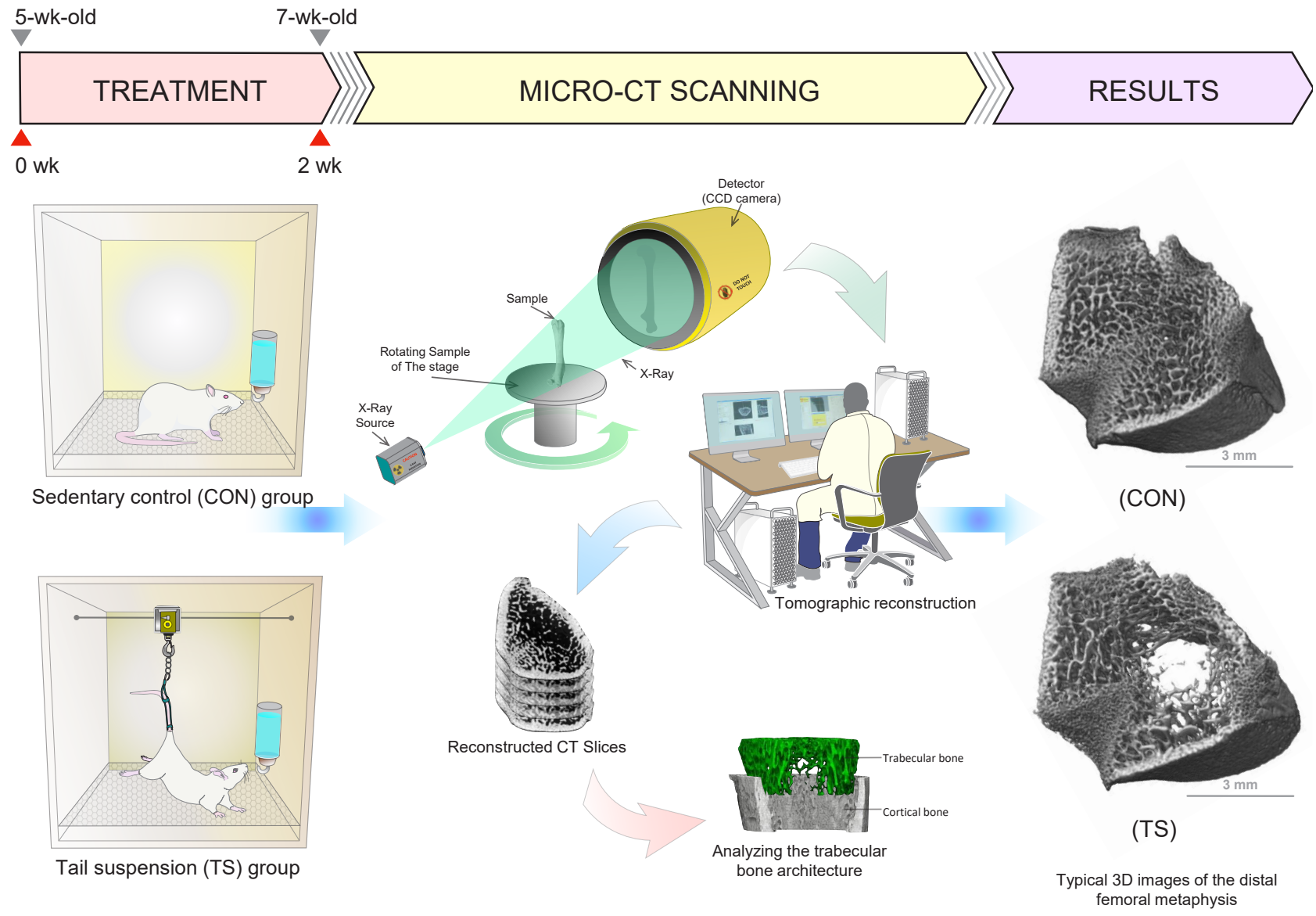

Fig. 1. Effects of hindlimb unloading on trabecular bone microarchitecture in rats. Skeletal unloading was simulated using the tail-suspended rat model. The 5-week-old male Wistar rats completed tail suspension for 2 weeks and were compared with sedentary control rats. After tail suspension for 2 weeks, to assess the 3-dimensional (3D) architecture of trabecular bone, the distal femurs were scanned using micro-computed tomography (micro-CT) and analyzed using 3D image analysis software. The tail-suspended rats exhibited a loss of trabeculae, particularly from the central zone of the femur with a loss of trabecular number, although the remaining trabeculae did not show significant differences in thickness compared to sedentary control rats.

apparent trabecular bone volume, $21 \%$ and $20 \%$ lower apparent trabecular number, respectively, compared to ablebodied men.

\section{EFFECTS OF TREADMILL RUNNING EXERCISE ON TRABECULAR BONE MICROARCHITECTURE}

Both treadmill and wheel-running exercise modalities in animal models are widely used to study the physiological adaptations associated with aerobic exercise.[23-27] In particular, weight-bearing exercises such as running on a treadmill have most commonly been utilized in animal models related to bone health, as the mechanisms involved are basically comparable to those in humans running on a treadmill.[28,29] The great advantage of using treadmill running is the availability of a variety of experimental protocols by strictly controlling exercise parameters such as treadmill speed, duration, frequency, angle, and additional weight, and also the ability to set precise exercise loads. However, a wide variety of protocols for treadmills (intensity, speed, duration, inclination, frequency, time) may be reflected in different magnitudes and rates of strain on bone while exercising. As a consequence, different treadmill protocols that are used in growing, young adult, ovariectomized (OVX), and osteopenia rats could have different effects on the trabecular bone structure. In experimental animal research, numerous studies have highlighted the fact that treadmill running exercise has been demonstrated to have positive effects on the microarchitecture of the 
femur and/or tibia coupled with an increase in trabecular parameters (i.e., trabecular bone volume fraction, trabecular number or trabecular thickness) of the tibia or distal femoral metaphysis.[11,30-36] In contrast to those results, treadmill running exercise does not always improve the microarchitecture. Indeed, some studies have suggested that the microarchitecture of the femur and/or tibia is not modified by treadmill running exercise.[37-42] In the worst cases, running exercise even induced deterioration of trabecular parameters in the tibial proximal epiphyses and/or thoracic vertebrae.[43-45] These differences in results have been explained by methodological inconsistencies inherent to animal protocols. Some studies have reported the relationship between exercise intensity and bone mass. Overly intense running exercise ( $>80 \%$ of $\mathrm{VO}_{2}$ max) reduces longitudinal bone growth and increases bone loss in rats,[30,43] while overly low intensity $\left(<40 \%\right.$ of $\mathrm{VO}_{2}$ max) may not provide sufficient mechanical stimulation for bone. [46] Accordingly, exercise intensities below $40 \% \mathrm{VO}_{2}$ max or above $80 \% \mathrm{VO}_{2}$ max are recognized as not achieving improvements to bone loss in rats.[30]

A study focusing on changes to the bone microarchitecture induced by treadmill running exercise in rats was first described in 1993 by Yeh et al. [11] In that histomorphometric study, they reported that the trabecular number in the tibial metaphysis was increased in 14-month-old Sprague-Dawley (SD) rats after 16 weeks of treadmill running exercise ( $20 \mathrm{~m} / \mathrm{min}, 60 \mathrm{~min} /$ day, 5 days/week), but trabecular thickness was unaffected. Iwamoto et al. [47] also showed that treadmill exercise $(30 \mathrm{~m} / \mathrm{min}, 60 \mathrm{~min} /$ day, 5 days/week for 12 weeks) in 23-week-old OVX Wistar rats improved cancellous bone mass in the proximal tibia as a result of increases in trabecular number (73\%) without altering trabecular thickness. Yao et al. [33] demonstrated that treadmill exercise $(12 \mathrm{~m} / \mathrm{min}, 90 \mathrm{~min} /$ day, 5 days/week for 5 weeks) in 10-week-old male Wistar rats induced a $22 \%$ increase in trabecular number and a 13\% increase in trabecular thickness in the tibial metaphysis compared with a
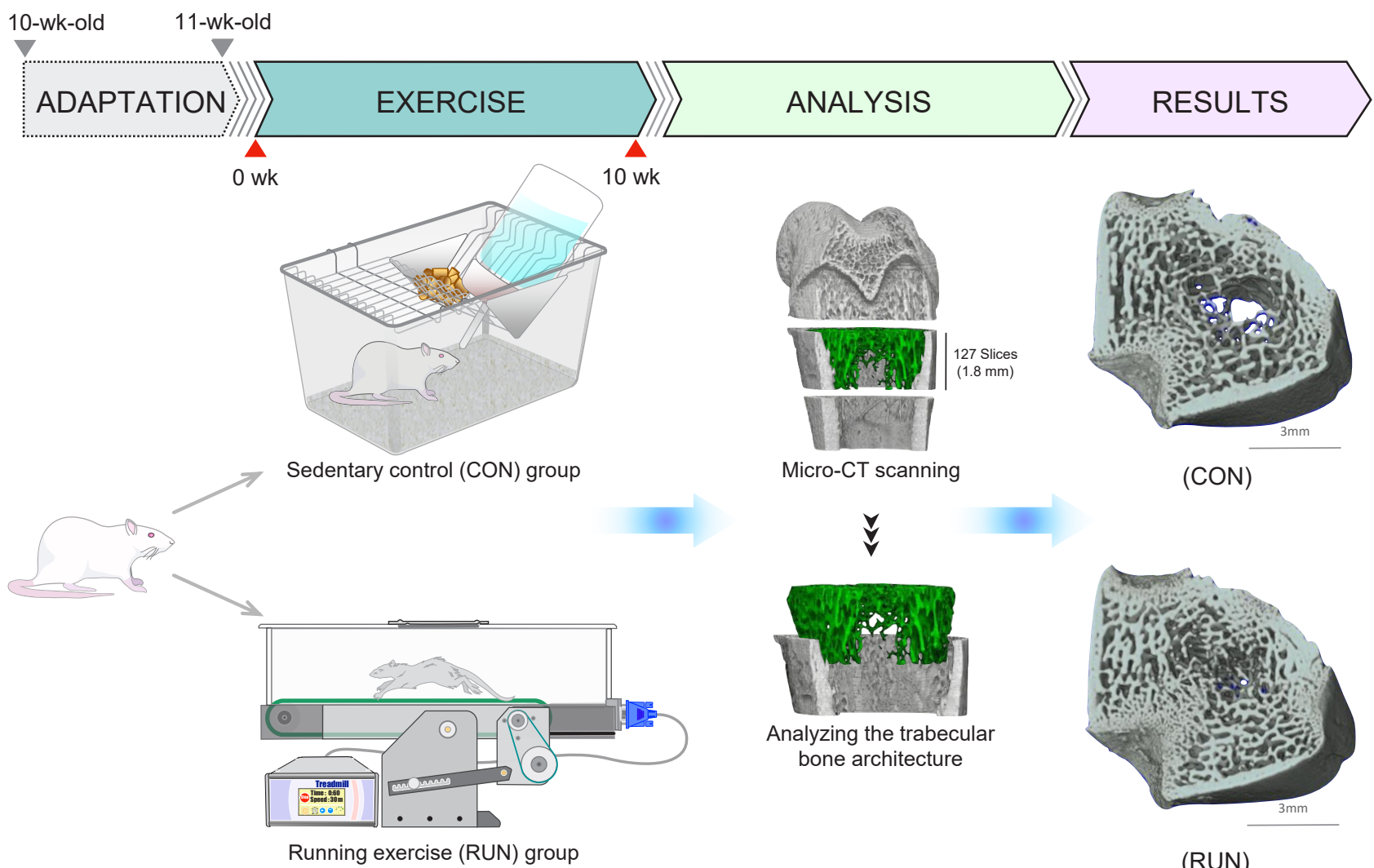
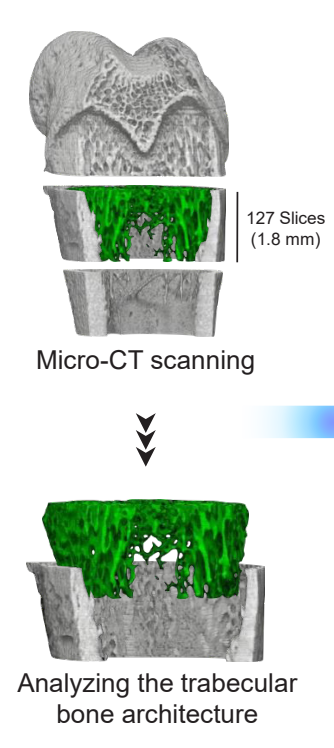

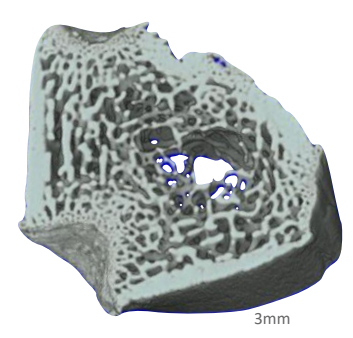

$(\mathrm{CON})$

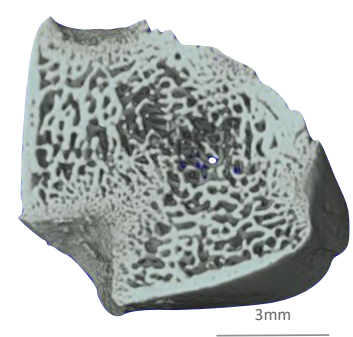

$(\mathrm{RUN})$

Fig. 2. Effects of treadmill running exercise on trabecular bone microarchitecture in rats. The 11-week-old male Wistar rats performed the treadmill running exercise of $30 \mathrm{~m} / \mathrm{min}$ for $60 \mathrm{~min}, 5$ times a week for 10 weeks. The 3-dimensional (3D) trabecular bone microarchitecture was evaluated at the distal femoral metaphysis using micro-computed tomography. Treadmill running exercise increased cancellous bone mass in the distal femurs, primarily by increasing trabecular number in growing rats. 
sedentary control group. The results of this study were similar to those in our previous experiments with 3D micro-CT of the distal femoral metaphysis, which showed that the increase in trabecular bone mass induced by moderate treadmill exercise $(30 \mathrm{~m} / \mathrm{min}, 60 \mathrm{~min} /$ day, 5 days/ week for 10 weeks) in 10-week-old Wistar rats was predominantly attributable to increases in trabecular number (22\%), with a slight increase in trabecular thickness (8\%) (Fig. 2).[31] We also showed that treadmill running exercise ( $25 \mathrm{~m} / \mathrm{min}, 60 \mathrm{~min} /$ day, 5 days/week) during the remobilization period after suspension-induced osteopenia induced significant increases in trabecular bone mass, primarily by significant alterations in trabecular number.[48] Moreover, this finding of a predominant effect of running exercise on trabecular microarchitecture agrees with the data of Berman et al. [14], who observed that the increase in cancellous bone mass due to treadmill running $(12 \mathrm{~m} / \mathrm{min}, 30$ $\mathrm{min} /$ day, 5 days/week, $5^{\circ}$ incline) for 6 weeks was predominately driven by increases in trabecular number rather than the trabecular thickness of the tibial metaphysis in 8-week-old male C57B1/6J mice. Taken together, such results suggest that, in the context of growing rats and mice, moderate treadmill running exercise increases cancellous bone mass as a result of increasing growth plate-derived new bone formation, although this is not uniformly the case.[49]

\section{EFFECTS OF JUMPING EXERCISE ON TRABECULAR BONE MICROARCHITECTURE}

Among the various types of loading, high-impact loading is considered particularly beneficial for bones compared to low- or moderate-impact loading. Jumping exercise is the most effective physical activity to provoke an osteogenic response due to the high tension and deformity applied to the skeleton.[50,51] Upward jumping in the form of vertical jumps from the bottom to the top of a board, without GRF on landing, is widely applied as a highimpact loading protocol for promoting increases in bone mass, bone strength, bone metabolism and structure in rats.[7,13,19,48,51-58] Some other studies have used jump resistance training (squat-like), $[6,40]$ free-fall impact exercise,[59-61] isometric strength training [62] or tower climbing, $[12,63]$ and each of these methods has proven effec- tive. An upward jump protocol is conducted by placing the rat inside a special wooden box surrounded by boards. Each rat has to jump, grasp the top of the board with the forelimbs, and climb up the board. The rat is then returned to the floor of the box to repeat the procedure. These works have demonstrated that the upward jump protocol has beneficial effects on bone mass, strength, and morphometry parameters, irrespective of animal sex or experimental model (young, adult, old, and osteopenia).[7,13,19,48,5158] Several previous histomorphometric analyses have found that the increase in trabecular bone mass achieved by jumping exercise is primarily due to increased trabecular thickness rather than to noticeable changes in numbers of trabeculae. $[6,13]$ In histomorphometric studies, Swift et al. [64] showed that trabecular thickness in the proximal tibial metaphysis was significantly increased following 5 weeks of jump resistance exercise (15 training sessions; 3 sessions/week interspersed by at least $48 \mathrm{hr}$ of rest) in 24week-old SD rats. Notomi et al. [6] found that jump resistance training (10 sets, 15 times/day for 8 weeks) in 8-weekold SD rats induced a $13 \%$ increase in trabecular thickness within the lumbar vertebrae without marked changes in trabecular number. They also reported that trabecular thickness in the lumbar vertebrae increased significantly by $12 \%$ following 4 weeks of jump resistance exercise (10 sets, 15 times/day) in 4-week-old SD rats, but the number of trabeculae was unaffected.[40]

Several micro-CT studies have found that the increase in trabecular bone mass by upward jump exercise is primarily attributable to increased trabecular thickness rather than noticeable changes in trabecular number.[13,48,64] We demonstrated that upward jump exercise (10 jumps/day, 5 days/week with a 40-cm jump height for 5 weeks) during the remobilization period after suspension-induced osteopenia ( 2 weeks) induced significant increases in trabecular thickness of $63 \%$ and trabecular number of $31 \%$ when compared with the spontaneous recovery group, resulting in a total increase in cancellous bone mass of the distal femoral metaphysis (Fig. 3).[48] We also reported that upward jump exercise (30 jumps/day, 5 days/week with a 40$\mathrm{cm}$ jump height) applied during hindlimb unloading (3 weeks) can prevent the microarchitectural deterioration of trabecular bone by increasing trabecular thickness while controlling the reduction of trabecular number in the distal femoral metaphysis.[65] Moreover, we showed that up- 


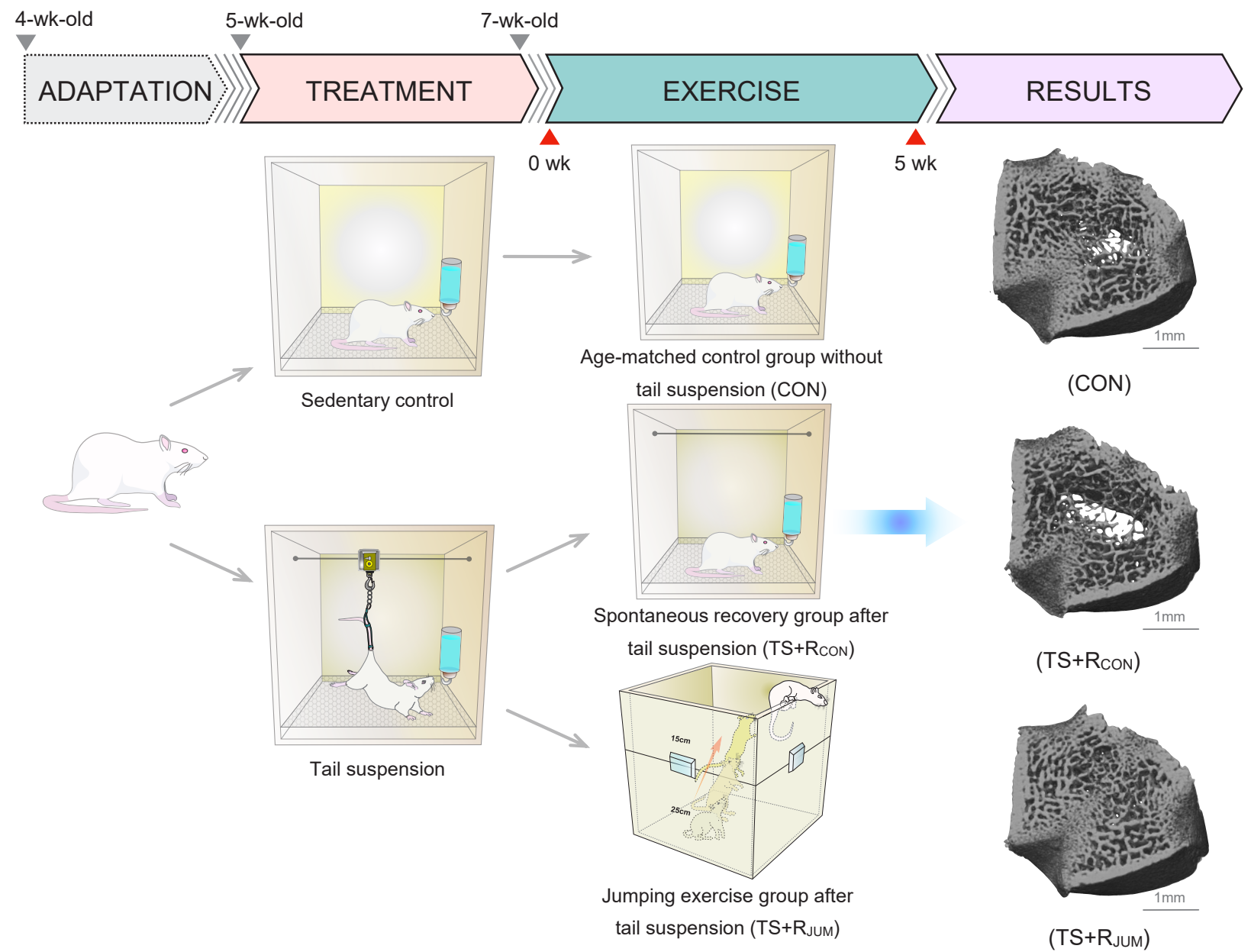

Fig. 3. Effects of jumping exercise on trabecular bone microarchitecture after tail suspension in rats. Tail suspension was conducted when rats were 5 weeks old, for 2 weeks. After removal from the tail suspension apparatus, rats jumped upwards 10 times/day, 5 days/week for 5 weeks with a jumping height of $40 \mathrm{~cm}$ and were compared with spontaneous recovery rats. The 5 weeks of spontaneous recovery after 2 weeks of skeletal unloading by tail suspension did not sufficiently recover femoral trabecular architecture in young growing rats. On the other hand, subsequent jumping exercise after tail suspension completely restored the deteriorated bone microarchitecture with a subsequent increase in trabecular thickness. [Modified from "Differential effects of jump versus running exercise on trabecular architecture during remobilization after suspension-induced osteopenia in growing rats.", by Ju YI, et al., 2012, J Appl Physiol (1985), 112, pp. 766-772. Copyright 2012 by the American Physiological Society. Reprinted with permission].

ward jump exercises (10 jumps/day, 5 days/week with a 40-cm jump height for 8 weeks) in 10-week-old Wistar rats induced significant increases in trabecular thickness of $51 \%$ and the trabecular number of $14 \%$ when compared with a sedentary control group.[13] The cancellous bone gain induced by upward jump exercise was suggested to be predominantly attributable to increases in trabecular thickness, with a slight increase in trabecular number. Similar findings have been confirmed in rats subjected to tower climbing.[12,63]

For the first time, we compared the effects of drop jump (10 drop jumps/day, 5 days/week for 8 weeks to heights of
40 and $60 \mathrm{~cm}$ ) and upward jump (10 upward jumps/day, 5 days/week for 8 weeks to a height of $40 \mathrm{~cm}$ ) impact trabecular bone mass and microarchitecture of the distal femur in growing rats.[66] We designed a specific device to ensure that rats landed on their hindlimbs during drop jumps to compare the effects of upward and drop jump impacts at the same skeletal site. We showed that trabecular bone mass in growing rats increased more effectively from the takeoff phases than from the landing phases of jumps, suggesting that the concentric muscle contraction of an upward jump would be more effective for stimulating trabecular bone mass than the eccentric muscle con- 
traction of a drop jump. We found that trabecular bone mass and thickness at the distal femur in growing rats significantly increased only with the upward jump exercise, compared with the drop jump exercise $(40$ and $60 \mathrm{~cm}$ ) and a sedentary control group. Drop jump exercise did not significantly improve trabecular bone mass except in trabecular number, despite a higher GRF. These results imply that the concentric muscle contraction from an upward jump would be more effective for stimulating elevations in trabecular bone mass than the eccentric muscle contraction of a drop jump. On the basis of these results, one of the characteristics of jumping exercise is that the change in trabecular bone microstructure is primarily due to the thickening of the trabecular bone.

\section{EFFECTS OF SWIMMING EXERCISE ON TRABECULAR BONE MICROARCHITECTURE}

Exercise is generally accepted as a key strategy to prevent osteoporosis and bone loss associated with aging. In particular, weight-bearing exercises such as running [67] or jumping $[68,69]$ are effective for enhancing bone mass. However, weight-bearing exercise may lead to an increased risk of stress fractures and osteoarthritis among elderly subjects with decreased physical function and fitness [70] and is thus unsuitable in exercise programs for elderly individuals, particularly for subjects displaying back and/or joint pain. Conversely, swimming, as a non-weight-bearing exercise, might be advantageous in exercise programs for elderly individuals since this activity is not accompanied by gravitational or impact stressors acting on weight-bearing bones. However, unlike running or jumping exercises, swimming exercise is not particularly suitable as an exercise protocol for increasing bone mass because the load on the skeleton is small. Swimmers training in this nonweight-bearing environment has therefore been shown to gain less skeletal benefit than athletes participating in weight-bearing activities.[2,5] In contrast to human swimmers, several studies examining the effects of swimming exercise on bone mass in the long bones of rats have shown that swimming exerts positive influences on bone mass in young,[71] adult,[72] tail-suspended,[73] OVX,[74,75] and high-fat diet-fed rats.[76,77] This discrepancy in the effects of swimming exercise on bone mass between human and animal studies demonstrated in the literature has created a great deal of confusion among researchers in this field. Given the lack of consensus on this topic, the precise effects of swimming on bone remain inconclusive.

In animal studies, swimming protocols are usually conducted by placing the animal inside a water bath maintained at 35 to $36^{\circ} \mathrm{C}$. Throughout the swimming exercise, the animal cannot touch the bottom or hang onto the sidewall of the barrel. Kang et al. [76] reported that swimming exercise (60 min/day, 5 times/week for 8 weeks) in SD rat models of high-fat diet-induced osteoporosis could prevent the trabecular architecture in the femur and tibia by increases in trabecular bone mass and trabecular number. Moreover, they found that swimming exercise $(60 \mathrm{~min} /$ day, 5 times/week for 8 weeks) in SD rats with high-fat diet-induced obesity improved the trabecular bone mass (60$80 \%$, respectively) of the femur and tibia as a result of increased trabecular number (50-58\%, respectively) and trabecular thickness (10-16\%, respectively).[77] Falcai et al. [78] also compared the effects of swimming (60 $\mathrm{min} /$ day, 5 days/week for 3 weeks), jumping (20 jumps/day, 5 days/ week for 3 weeks), and vibration therapies (20 min, 5 days/ week for 3 weeks; longitudinal amplitude, $1 \mathrm{~mm}$; frequen$\mathrm{cy}, 50 \mathrm{~Hz} /$ day) on the prevention of micro-architectural deterioration of bone using a rat model with hindlimb unloading. They demonstrated that swimming showed similar levels of osteogenic effect to jumping and a slightly higher effect than a vibration. Moreover, swimming exercise has been reported to produce greater bone adaptations than running in rapidly growing female rats.[79] Furthermore, we examined whether swimming (60 min/day, 5 days/week for 12 weeks) exerts beneficial effects on preventing the loss of trabecular bone mass and the deterioration of trabecular microarchitecture in the femur in a rat model of postmenopausal osteoporosis.[75] In this study, swimming-exercised OVX rats showed a $102 \%$ increase in trabecular bone mass, $71 \%$ increase in trabecular number, and $18 \%$ increase in thickness compared with OVX sedentary control rats. In sham-treated rats, swimming exercise also induced significant increases in trabecular bone mass (64\%), trabecular number (42\%), and thickness (16\%) when compared with sedentary control rats (Fig. 4). Interestingly, the increased rate of gain in metaphyseal trabecular bone mass through swimming exercise in this study resembled that in our previous experiment,[19] which showed that jumping exercise during the remobilization period induced 


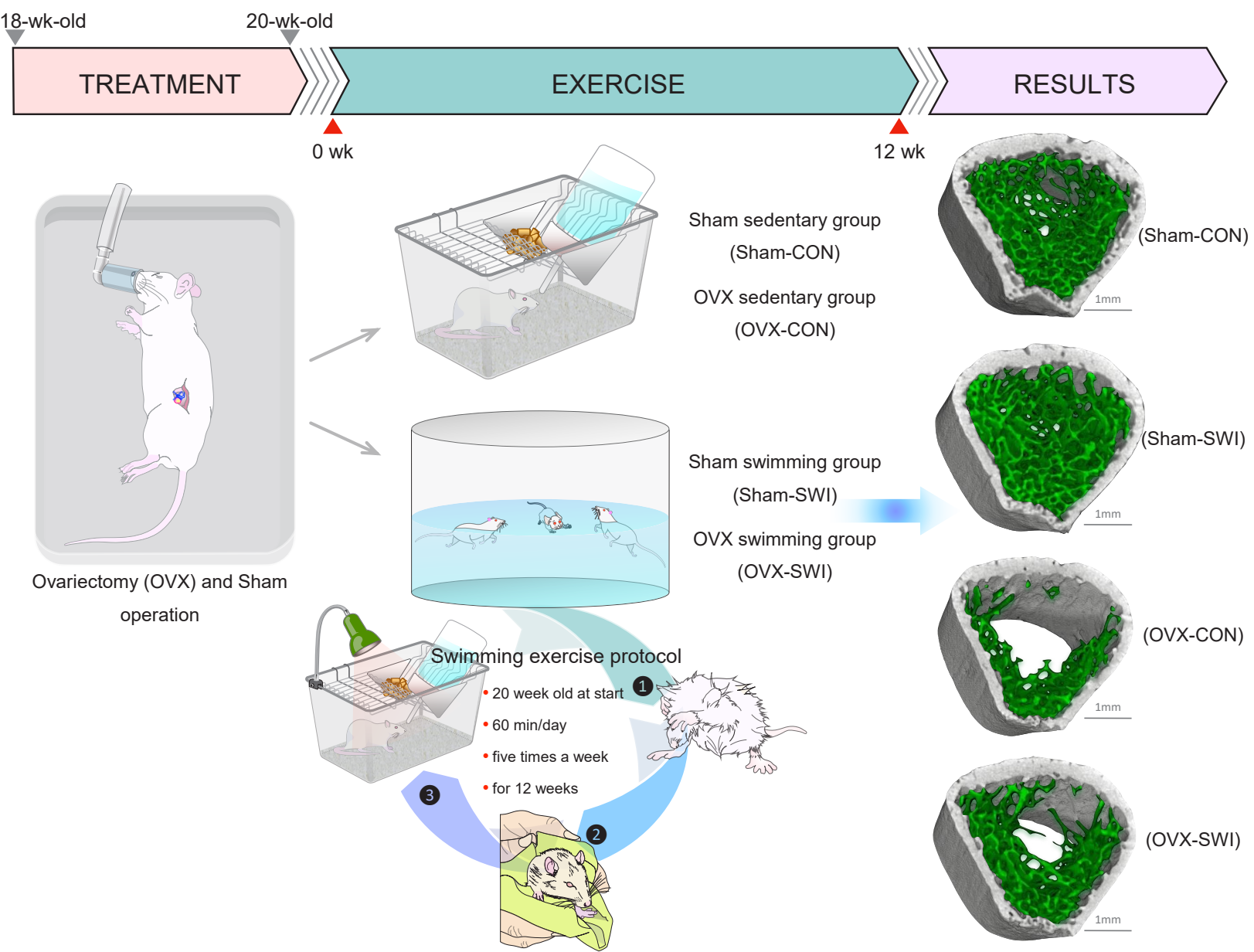

Fig. 4. Effects of swimming exercise on trabecular bone microarchitecture in ovariectomized rats. Ovariectomies and sham operations were conducted on 18-week-old female rats. After postoperative recovery for 2 weeks after the operation, rats performed swimming in a water bath for 60 $\mathrm{min} /$ day, 5 days/week, for 12 weeks. Trabecular bone thickness and number in the distal femoral metaphysis were decreased by ovariectomy. On the other hand, these changes were suppressed by swimming exercise. In sham-treated rats, swimming exercise also induced a significant increase in trabecular bone mass, primarily via significant alterations in trabecular number. [Modified from "Effect of swimming exercise on 3-dimensional trabecular bone microarchitecture in ovariectomized rats.", by Ju Yl, et al., 2015, J Appl Physiol (1985), 119, pp. 990-997. Copyright 2015 by the American Physiological Society. Reprinted with permission].

significant increases in trabecular bone mass by $64 \%$, trabecular number by $31 \%$, and trabecular thickness by $63 \%$ when compared with spontaneous recovery rats. These results support previous studies showing that the percentage increase in trabecular bone mass for the proximal tibia gained through swimming exercise was similar to values measured in jumping-exercised rats.[78] These results, as a whole, provide additional support for the oft-mentioned notion that swimming exercise may be beneficial for improving or attenuating losses in bone mass and structure in small animals.

The osteogenic effect observed in swim-exercised rats is derived primarily from the mechanical loading generated by muscle contraction. This implies that in small laboratory animals such as rats, muscle contraction during swimming may provide levels of mechanical stress comparable to those observed in jumping exercise and even higher levels than running exercise. This contrasts sharply with crosssectional studies that have demonstrated a lower bone mass in swimmers than athletes engaged in weight-bearing and impact sports. Direct extrapolation of results from rats to humans is thus inappropriate. However, not all swimming studies in animals have been associated with bone gains.[80-85] Bourrin et al. [81] reported that swimming exercise for prolonged periods in growing rats exerted negative effects on both trabecular number and thickness 
Table 1. Summary on the effects of different types of mechanical loading on trabecular bone microarchitecture in rats

\begin{tabular}{|c|c|c|c|}
\hline Treatment & Skeletal sites & Analysis & Key findings \\
\hline $\begin{array}{l}\text { Skeletal unloading by tail } \\
\text { suspension }\end{array}$ & Tibia, femur & Histomorphometry, micro-CT & $\begin{array}{l}\text { - Significant decrease in the number of trabeculae } \\
\text { - No marked changes in trabecular thickness }\end{array}$ \\
\hline Running exercise & Tibia, femur & Histomorphometry, micro-CT & $\begin{array}{l}\text { - Significant increase in the number of trabeculae } \\
\text { - Slight increase in trabecular thickness }\end{array}$ \\
\hline Jump exercise & $\begin{array}{l}\text { Tibia, lumbar vertebra, } \\
\text { femur }\end{array}$ & Histomorphometry, micro-CT & $\begin{array}{l}\text { - Marked thickening of the trabeculae } \\
\text { - Slight increase or no marked changes in trabecular number }\end{array}$ \\
\hline Swimming exercise & Tibia, femur & Histomorphometry, micro-CT & $\begin{array}{l}\text { - Significant increase in the number of trabeculae } \\
\text { - Slight increase in trabecular thickness }\end{array}$ \\
\hline
\end{tabular}

CT, computed tomography.

of the distal femoral metaphysis. The exercise protocol used by Bourrin et al. [81] comprised $6 \mathrm{hr} /$ day of swimming, 5 days/week at the end of the experiment, roughly comparable to the regimen of collegiate swimmers at the peak of the training season. The reason for this discrepancy between different studies remains unclear but may be due to differences in duration of swimming exercises performed by rats. Therefore, even in rats, extended time exercising in a hypogravity environment may result in deleterious effects on bone, as in the case of humans. Further studies are needed to clarify the specific effects of swimming exercise on bone structure in humans and animals.

\section{CONCLUSIONS}

Based on the literature discussed in this review, we concluded that treadmill running, jumping, and swimming exercises increased trabecular bone mass in small animals and interestingly, exerted different effects on the trabecular bone microarchitecture. That is, low-impact loading such as running and swimming exercises increased trabecular bone mass specifically by increasing the number of trabeculae with slight increases in trabecular thickness. In contrast to treadmill running and swimming exercises, high-impact loading such as jumping exercise increased trabecular bone mass by thickening trabeculae. We provide a brief summary in Table 1 based on our latest research and previous research findings. Collectively, these results suggest that treadmill running, jumping, and swimming exercises have different mechanisms of action on the structural characteristics of trabecular bone in rats. The reason for this phenomenon is unclear, but different patterns of loading stress (strain rate, strain magnitude, cycle number, loading direction, etc.) might result in different patterns of reaction in the trabecular architecture. The effect (if any) of these structural differences on bone strength deserves further study.

\section{DECLARATIONS}

\section{Ethics approval and consent to participate Not applicable.}

\section{Conflict of interest}

No potential conflict of interest relevant to this article was reported.

\section{ORCID}

Yong-In Ju https://orcid.org/0000-0001-8653-7623

Teruki Sone https://orcid.org/0000-0002-3257-4063

\section{REFERENCES}

1. Bassey EJ, Ramsdale SJ. Weight-bearing exercise and ground reaction forces: a 12-month randomized controlled trial of effects on bone mineral density in healthy postmenopausal women. Bone 1995;16:469-76. https://doi.org/10.1016/ 8756-3282(95)90193-0.

2. Duncan CS, Blimkie CJ, Kemp A, et al. Mid-femur geometry and biomechanical properties in 15- to 18-yr-old female athletes. Med Sci Sports Exerc 2002;34:673-81. https: //doi.org/10.1097/00005768-200204000-00018.

3. Gruodyte R, Jürimäe J, Cicchella $A$, et al. Adipocytokines and bone mineral density in adolescent female athletes. Acta Paediatr 2010;99:1879-84. https://doi.org/10.1111/ j.1651-2227.2010.01905.x.

4. Lee EJ, Long KA, Risser WL, et al. Variations in bone status of contralateral and regional sites in young athletic women. Med Sci Sports Exerc 1995;27:1354-61.

5. Taaffe DR, Snow-Harter C, Connolly DA, et al. Differential 
effects of swimming versus weight-bearing activity on bone mineral status of eumenorrheic athletes. J Bone Miner Res 1995;10:586-93. https://doi.org/10.1002/jbmr.5650100411.

6. Notomi T, Lee SJ, Okimoto N, et al. Effects of resistance exercise training on mass, strength, and turnover of bone in growing rats. Eur J Appl Physiol 2000;82:268-74. https:// doi.org/10.1007/s004210000195.

7. Umemura $\mathrm{Y}$, Ishiko $\mathrm{T}$, Tsujimoto $\mathrm{H}$, et al. Effects of jump training on bone hypertrophy in young and old rats. Int J Sports Med 1995;16:364-7. https://doi.org/10.1055/s-2007973021.

8. Judex S, Zernicke RF. Does the mechanical milieu associated with high-speed running lead to adaptive changes in diaphyseal growing bone? Bone 2000;26:153-9. https:// doi.org/10.1016/s8756-3282(99)00256-2.

9. Judex S, Zernicke RF. High-impact exercise and growing bone: relation between high strain rates and enhanced bone formation. J Appl Physiol (1985) 2000;88:2183-91. https://doi.org/10.1152/jappl.2000.88.6.2183.

10. Westerlind KC, Fluckey JD, Gordon SE, et al. Effect of resistance exercise training on cortical and cancellous bone in mature male rats. J Appl Physiol (1985) 1998;84:459-64. https://doi.org/10.1152/jappl.1998.84.2.459.

11. Yeh JK, Aloia JF, Chen MM, et al. Influence of exercise on cancellous bone of the aged female rat. J Bone Miner Res 1993;8:1117-25. https://doi.org/10.1002/jbmr.5650080913.

12. Notomi T, Okimoto N, Okazaki Y, et al. Effects of tower climbing exercise on bone mass, strength, and turnover in growing rats. J Bone Miner Res 2001;16:166-74. https://doi.org/ 10.1359/jbmr.2001.16.1.166.

13. Ju Yl, Choi HJ, Ohnaru K, et al. Differential effects of jump versus running exercise on trabecular bone architecture and strength in rats. Phys Act Nutr 2020;24:1-8. https:// doi.org/10.20463/pan.2020.0001.

14. Berman AG, Hinton MJ, Wallace JM. Treadmill running and targeted tibial loading differentially improve bone mass in mice. Bone Rep 2019;10:100195. https://doi.org/10.1016/ j.bonr.2019.100195.

15. Morey ER, Sabelman EE, Turner RT, et al. A new rat model simulating some aspects of space flight. Physiologist 1979; 22:S23-4.

16. Martin RB. Effects of simulated weightlessness on bone properties in rats. J Biomech 1990;23:1021-9. https://doi. org/10.1016/0021-9290(90)90317-v.

17. Wronski TJ, Morey-Holton ER. Skeletal response to simu- lated weightlessness: a comparison of suspension techniques. Aviat Space Environ Med 1987;58:63-8.

18. Basso N, Bellows CG, Heersche JN. Effect of simulated weightlessness on osteoprogenitor cell number and proliferation in young and adult rats. Bone 2005;36:173-83. https: //doi.org/10.1016/j.bone.2004.09.016.

19. Ju Yl, Sone T, Okamoto T, et al. Jump exercise during remobilization restores integrity of the trabecular architecture after tail suspension in young rats. J Appl Physiol (1985) 2008;104:1594-600. https://doi.org/10.1152/japplphysiol.01004.2007.

20. Bourrin S, Palle S, Genty C, et al. Physical exercise during remobilization restores a normal bone trabecular network after tail suspension-induced osteopenia in young rats. $J$ Bone Miner Res 1995;10:820-8. https://doi.org/10.1002/ jbmr.5650100520.

21. Armbrecht G, Belavý DL, Backström M, et al. Trabecular and cortical bone density and architecture in women after 60 days of bed rest using high-resolution pQCT: WISE 2005. J Bone Miner Res 2011;26:2399-410. https://doi. org/10.1002/jbmr.482.

22. Modlesky CM, Majumdar S, Narasimhan A, et al. Trabecular bone microarchitecture is deteriorated in men with spinal cord injury. J Bone Miner Res 2004;19:48-55. https: //doi.org/10.1359/jbmr.0301208.

23. Allen JM, Berg Miller ME, Pence BD, et al. Voluntary and forced exercise differentially alters the gut microbiome in C57BL/6J mice. J Appl Physiol (1985) 2015;118:1059-66. https://doi.org/10.1152/japplphysiol.01077.2014.

24. Eadie BD, Redila VA, Christie BR. Voluntary exercise alters the cytoarchitecture of the adult dentate gyrus by increasing cellular proliferation, dendritic complexity, and spine density. J Comp Neurol 2005;486:39-47. https://doi.org/ 10.1002/cne.20493.

25. Kang C, Chung E, Diffee G, et al. Exercise training attenuates aging-associated mitochondrial dysfunction in rat skeletal muscle: role of PGC-1a. Exp Gerontol 2013;48:134350. https://doi.org/10.1016/j.exger.2013.08.004.

26. Roemers P, Mazzola PN, De Deyn PP, et al. Burrowing as a novel voluntary strength training method for mice: A comparison of various voluntary strength or resistance exercise methods. J Neurosci Methods 2018;300:112-26. https: //doi.org/10.1016/j.jneumeth.2017.05.027.

27. Seldeen KL, Lasky G, Leiker MM, et al. High intensity interval training improves physical performance and frailty in 
aged mice. J Gerontol A Biol Sci Med Sci 2018;73:429-37. https://doi.org/10.1093/gerona/glx120.

28. Bozzini C, Viscor G, Kristjánsson JCR, et al. Increased intrinsic stiffness and mineralization in femurs of adult rats after high intensity treadmill running training. Biomed Mater Eng 2017;28:431-41. https://doi.org/10.3233/bme-171684.

29. Huang TH, Su IH, Lewis JL, et al. Effects of methionine restriction and endurance exercise on bones of ovariectomized rats: a study of histomorphometry, densitometry, and biomechanical properties. J Appl Physiol (1985) 2015; 119:517-26. https://doi.org/10.1152/japplphysiol.00395. 2015.

30. Bourrin S, Palle S, Pupier R, et al. Effect of physical training on bone adaptation in three zones of the rat tibia. J Bone Miner Res 1995;10:1745-52. https://doi.org/10.1002/jbmr. 5650101118.

31. Joo Yl, Sone T, Fukunaga $M$, et al. Effects of endurance exercise on three-dimensional trabecular bone microarchitecture in young growing rats. Bone 2003;33:485-93. https: //doi.org/10.1016/s8756-3282(03)00212-6.

32. Yeh JK, Aloia JF, Tierney JM, et al. Effect of treadmill exercise on vertebral and tibial bone mineral content and bone mineral density in the aged adult rat: determined by dual energy X-ray absorptiometry. Calcif Tissue Int 1993;52:2348. https://doi.org/10.1007/bf00298725.

33. Yao Z, Lafage-Proust MH, Plouët J, et al. Increase of both angiogenesis and bone mass in response to exercise depends on VEGF. J Bone Miner Res 2004;19:1471-80. https: //doi.org/10.1359/jbmr.040517.

34. Iwamoto J, Shimamura C, Takeda T, et al. Effects of treadmill exercise on bone mass, bone metabolism, and calciotropic hormones in young growing rats. J Bone Miner Metab 2004;22:26-31. https://doi.org/10.1007/s00774-003-0443-5.

35. Chen Y, Wang S, Bu S, et al. Treadmill training prevents bone loss by inhibition of PPARy expression but not promoting of Runx2 expression in ovariectomized rats. Eur J Appl Physiol 2011;111:1759-67. https://doi.org/10.1007/ s00421-010-1820-0.

36. Iwamoto J, Yeh JK, Aloia JF. Differential effect of treadmill exercise on three cancellous bone sites in the young growing rat. Bone 1999;24:163-9. https://doi.org/10.1016/s87563282(98)00189-6.

37. Hamann N, Kohler T, Müller R, et al. The effect of level and downhill running on cortical and trabecular bone in growing rats. Calcif Tissue Int 2012;90:429-37. https://doi.org/ 10.1007/s00223-012-9593-6.

38. Leppänen OV, Sievänen $\mathrm{H}$, Jokihaara J, et al. Pathogenesis of age-related osteoporosis: impaired mechano-responsiveness of bone is not the culprit. PLoS One 2008;3:e2540. https://doi.org/10.1371/journal.pone.0002540.

39. Tromp AM, Bravenboer N, Tanck E, et al. Additional weight bearing during exercise and estrogen in the rat: the effect on bone mass, turnover, and structure. Calcif Tissue Int 2006;79:404-15. https://doi.org/10.1007/s00223-006-0045-z.

40. Notomi T, Okazaki Y, Okimoto $\mathrm{N}$, et al. A comparison of resistance and aerobic training for mass, strength and turnover of bone in growing rats. Eur J Appl Physiol 2000;83: 469-74. https://doi.org/10.1007/s004210000316.

41. Maurel DB, Boisseau N, Ingrand I, et al. Combined effects of chronic alcohol consumption and physical activity on bone health: study in a rat model. Eur J Appl Physiol 2011; 111:2931-40. https://doi.org/10.1007/s00421-011-1916-1.

42. Reed AH, McCarty HL, Evans GL, et al. The effects of chronic alcohol consumption and exercise on the skeleton of adult male rats. Alcohol Clin Exp Res 2002;26:1269-74. https://doi.org/10.1097/01.Alc.0000023984.47311.6e.

43. Bourrin S, Genty C, Palle S, et al. Adverse effects of strenuous exercise: a densitometric and histomorphometric study in the rat. J Appl Physiol (1985) 1994;76:1999-2005. https://doi.org/10.1152/jappl.1994.76.5.1999.

44. Maurel DB, Boisseau N, Pallu S, et al. Regular exercise limits alcohol effects on trabecular, cortical thickness and porosity, and osteocyte apoptosis in the rat. Joint Bone Spine 2013;80:492-8. https://doi.org/10.1016/j.jbspin.2012.12.005.

45. Sipos W, Rauner M, Skalicky M, et al. Running has a negative effect on bone metabolism and proinflammatory status in male aged rats. Exp Gerontol 2008;43:578-83. https: //doi.org/10.1016/j.exger.2008.03.008.

46. Bourrin S, Zerath E, Vico L, et al. Bone mass and bone cellular variations after five months of physical training in rhesus monkeys: histomorphometric study. Calcif Tissue Int 1992;50:404-10. https://doi.org/10.1007/bf00296770.

47. Iwamoto J, Takeda T, Ichimura S. Effects of moderate intensity exercise on tibial bone mass in mature ovariectomized rats: bone histomorphometry study. Keio J Med 1998;47:162-7. https://doi.org/10.2302/kjm.47.162.

48. Ju YI, Sone T, Ohnaru K, et al. Differential effects of jump versus running exercise on trabecular architecture during remobilization after suspension-induced osteopenia in growing rats. J Appl Physiol (1985) 2012;112:766-72. https: 
//doi.org/10.1152/japplphysiol.01219.2011.

49. Liu Z, Gao J, Gong H. Effects of treadmill with different intensities on bone quality and muscle properties in adult rats. Biomed Eng Online 2019;18:107. https://doi.org/ 10.1186/s12938-019-0728-0.

50. Nikander R, Sievänen $H$, Heinonen $A$, et al. Targeted exercise against osteoporosis: A systematic review and metaanalysis for optimising bone strength throughout life. BMC Med 2010;8:47. https://doi.org/10.1186/1741-70158-47.

51. Umemura Y, Nagasawa S, Honda A, et al. High-impact exercise frequency per week or day for osteogenic response in rats. J Bone Miner Metab 2008;26:456-60. https://doi. org/10.1007/s00774-007-0848-7.

52. Nagasawa S, Umemura Y. Bone hypertrophy in rats: Effects of jump number and height. Adv Exerc Sports Physiol 2002; 8:87-92.

53. Honda A, Umemura Y, Nagasawa S. Effect of high-impact and low-repetition training on bones in ovariectomized rats. J Bone Miner Res 2001;16:1688-93. https://doi.org/ 10.1359/jbmr.2001.16.9.1688.

54. Honda A, Sogo N, Nagasawa S, et al. High-impact exercise strengthens bone in osteopenic ovariectomized rats with the same outcome as Sham rats. J Appl Physiol (1985) 2003; 95:1032-7. https://doi.org/10.1152/japplphysiol.00781. 2002.

55. Honda A, Sogo N, Nagasawa S, et al. Bones benefits gained by jump training are preserved after detraining in young and adult rats. J Appl Physiol (1985) 2008;105:849-53. https: //doi.org/10.1152/japplphysiol.00902.2007.

56. Ooi FK, Singh R, Singh HJ, et al. Minimum level of jumping exercise required to maintain exercise-induced bone gains in female rats. Osteoporos Int 2009;20:963-72. https://doi. org/10.1007/s00198-008-0760-6.

57. Umemura $\mathrm{Y}$, Nagasawa $\mathrm{S}$, Sogo $\mathrm{N}$, et al. Effects of jump training on bone are preserved after detraining, regardless of estrogen secretion state in rats. J Appl Physiol (1985) 2008;104:1116-20. https://doi.org/10.1152/japplphysiol. 00937.2007.

58. Umemura Y, Ishiko T, Yamauchi T, et al. Five jumps per day increase bone mass and breaking force in rats. J Bone Miner Res 1997;12:1480-5. https://doi.org/10.1359/jbmr.1997. 12.9.1480.

59. Lin HS, Huang TH, Wang HS, et al. Short-term free-fall landing causes reduced bone size and bending energy in fem- ora of growing rats. J Sports Sci Med 2013;12:1-9.

60. Welch JM, Turner CH, Devareddy L, et al. High impact exercise is more beneficial than dietary calcium for building bone strength in the growing rat skeleton. Bone 2008;42: 660-8. https://doi.org/10.1016/j.bone.2007.12.220.

61. Welch JM, Weaver CM, Turner CH. Adaptations to free-fall impact are different in the shafts and bone ends of rat forelimbs. J Appl Physiol (1985) 2004;97:1859-65. https:// doi.org/10.1152/japplphysiol.00438.2004.

62. Cavalié H, Horcajada-Molteni MN, Lebecque P, et al. Progressive isometric force training and bone mass in rats. $J$ Musculoskelet Neuronal Interact 2003;3:47-52.

63. Notomi T, Okazaki Y, Okimoto N, et al. Effects of tower climbing exercise on bone mass, strength, and turnover in orchidectomized growing rats. J Appl Physiol (1985) 2002; 93:1152-8. https://doi.org/10.1152/japplphysiol.01221. 2001.

64. Swift JM, Gasier HG, Swift SN, et al. Increased training loads do not magnify cancellous bone gains with rodent jump resistance exercise. J Appl Physiol (1985) 2010;109:16007. https://doi.org/10.1152/japplphysiol.00596.2010.

65. Ju YI, Sone T, Ohnaru K, et al. Jump exercise during hindlimb unloading protect against the deterioration of trabecular bone microarchitecture in growing young rats. Springerplus 2013;2:35. https://doi.org/10.1186/2193-1801-2-35.

66. Ju Yl, Sone T, Ohnaru K, et al. Effects of different types of jump impact on trabecular bone mass and microarchitecture in growing rats. PLoS One 2014;9:e107953. https:// doi.org/10.1371/journal.pone.0107953.

67. Hawkins SA, Wiswell RA, Jaque SV, et al. The inability of hormone replacement therapy or chronic running to maintain bone mass in master athletes. J Gerontol A Biol Sci Med Sci 1999;54:M451-5. https://doi.org/10.1093/gerona/ 54.9.m451.

68. Winters KM, Snow CM. Detraining reverses positive effects of exercise on the musculoskeletal system in premenopausal women. J Bone Miner Res 2000;15:2495-503. https: //doi.org/10.1359/jbmr.2000.15.12.2495.

69. Witzke KA, Snow CM. Effects of plyometric jump training on bone mass in adolescent girls. Med Sci Sports Exerc 2000;32:1051-7. https://doi.org/10.1097/00005768-200 006000-00003.

70. Turner $\mathrm{CH}$. Exercise as a therapy for osteoporosis: the drunk and the street lamp, revisited. Bone 1998;23:83-5. https:// doi.org/10.1016/s8756-3282(98)00088-x. 
71. Simkin A, Leichter I, Swissa A, et al. The effect of swimming activity on bone architecture in growing rats. J Biomech 1989;22:845-51. https://doi.org/10.1016/0021-9290(89) 90068-7.

72. Swissa-Sivan A, Statter M, Brooks GA, et al. Effect of swimming on prednisolone-induced osteoporosis in elderly rats. J Bone Miner Res 1992;7:161-9. https://doi.org/10.1002/ jbmr.5650070207.

73. Falcai MJ, Zamarioli A, Leoni GB, et al. Swimming activity prevents the unloading induced loss of bone mass, architecture, and strength in rats. Biomed Res Int 2015;2015: 507848. https://doi.org/10.1155/2015/507848.

74. Hart KJ, Shaw JM, Vajda E, et al. Swim-trained rats have greater bone mass, density, strength, and dynamics. J Appl Physiol (1985) 2001;91:1663-8. https://doi.org/10.1152/ jappl.2001.91.4.1663.

75. Ju YI, Sone T, Ohnaru K, et al. Effect of swimming exercise on three-dimensional trabecular bone microarchitecture in ovariectomized rats. J Appl Physiol (1985) 2015;119: 990-7. https://doi.org/10.1152/japplphysiol.00147.2015.

76. Kang YS, Kim SH, Kim JC. Effects of swimming exercise on high-fat diet-induced low bone mineral density and trabecular bone microstructure in rats. J Exerc Nutrition Biochem 2017;21:48-55. https://doi.org/10.20463/jenb.2016. 0063.

77. Kang YS, Kim JC, Kim JS, et al. Effects of swimming exercise on serum irisin and bone FNDC5 in rat models of highfat diet-induced osteoporosis. J Sports Sci Med 2019;18: 596-603.

78. Falcai MJ, Zamarioli A, Okubo R, et al. The osteogenic effects of swimming, jumping, and vibration on the protec- tion of bone quality from disuse bone loss. Scand J Med Sci Sports 2015;25:390-7. https://doi.org/10.1111/sms. 12240.

79. Snyder A, Zierath JR, Hawley JA, et al. The effects of exercise mode, swimming vs. running, upon bone growth in the rapidly growing female rat. Mech Ageing Dev 1992; 66:59-69. https://doi.org/10.1016/0047-6374(92)90073-m.

80. Barreto TK, Bizarria FS, Coutinho MP, et al. Is swimming able to maintain bone health and to minimize postmenopausal bone resorption? Rev Bras Reumatol 2014;54:110-6.

81. Bourrin S, Ghaemmaghami F, Vico L, et al. Effect of a fiveweek swimming program on rat bone: a histomorphometric study. Calcif Tissue Int 1992;51:137-42. https://doi. org/10.1007/bf00298502.

82. Figard H, Mougin F, Gaume V, et al. Combined intervention of dietary soybean proteins and swim training: effects on bone metabolism in ovariectomized rats. J Bone Miner Metab 2006;24:206-12. https://doi.org/10.1007/s00774005-0673-9.

83. Hoshi $A$, Watanabe $H$, Chiba $M$, et al. Bone density and mechanical properties in femoral bone of swim loaded aged mice. Biomed Environ Sci 1998;11:243-50.

84. Hoshi A, Watanabe $H$, Chiba $M$, et al. Effects of swimming and weight loading on bone density and mechanical properties of the mouse femoral bone. Environ Health Prev Med 1996;1:128-32. https://doi.org/10.1007/bf02931203.

85. Park J, Omi N. The effects of different exercise modes for preventing endothelial dysfunction of arteries and bone loss in ovariectomized rats. J Exerc Nutrition Biochem 2014; 18:133-9. https://doi.org/10.5717/jenb.2014.18.2.133. 
